\title{
Solar active envelope module with an adjustable transmittance/absorptance
}

\author{
C. Villasante ${ }^{\mathrm{a}, *}$, I. del Hoyo ${ }^{\mathrm{a}}$, I. Pagola ${ }^{\mathrm{b}}$, M. Sánchez ${ }^{\mathrm{b}}$ and E. Aranzabe ${ }^{\mathrm{a}}$ \\ ${ }^{a}$ Mechanical Engineering Unit IK4-tekniker, C/Iñaki Goenaga, Eibar, Gipuzkoa, Spain

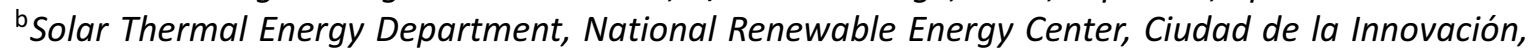 \\ Sarriguren (Navarra), España
}

\begin{abstract}
A solar active envelope module with a high flexibility degree is proposed in this paper. The transparent module controls the day-lighting of the room, improving the indoor environment, while absorbing the superfluous solar energy inside. That energy is used to increase the efficiency of heating, ventilation, and the air-conditioning (HVAC) system of the building. This is carried out through a fine control of the absorptance of the envelope module. The active envelope module consists of three glazed chambers with advanced coatings and frames to assure a minimum thermal transmittance while allowing transparency. A fluid containing heat-absorbing nanoparticles flows inside the central chamber and is heated up due to the impinging solar energy. Unlike other systems proposed in the past, which included transparency control systems based on complex filters and chemical processes, the absorption of the module is controlled by the variation of the thickness of the central chamber with a mechanical device. That is, varying the thickness of the central chamber, it allows controlling the absorptance of the whole system and, as a result, indoor day-lighting and thermal loads. Therefore, a new system is proposed that enables to:
\end{abstract}

- Dynamically control the thermal loads and solar day-lighting.

- Improve the thermal performance of building envelope and reduce the energy demand of the building.

- Increase the solar fraction of the heating and cooling, and reduce the thermal losses improving the efficiency of the building.

The solar active device proposed in this article is protected by a Spanish patent application (Fundación Tekniker, Fundación CENER-CIEMAT, 2014).

Keywords: Solar collector facade, architectural module, adaptive envelope, absorptance regulation, indoor comfort, dynamic control, thermal loads reduction

\section{Introduction}

Several facade solutions that control the indoor day lighting for an improved environment can be found in the literature. For instance, traditional blinds and shutters have been widely used and recently, products like Isolette ${ }^{\circledR}$ include built-in blinds. Some other existing modules, in addition of controlling the day-lighting through the facade, absorb the part of the solar energy that is not needed inside, and use it for different purposes, such as the increase of heating, ventilation and air-conditioning (HVAC) system of the building efficiency.

\footnotetext{
${ }^{*}$ Corresponding author: C. Villasante, Mechanical Engineering Unit IK4-tekniker, C/Iñaki Goenaga, 520600 Eibar, Gipuzkoa, Spain. Tel.: +34 943206744 / 9432569 00; Fax: +34 9432027 57; E-mail: cristobal.villasante@tekniker.es.
} 
Amongst these facade solutions that control the lighting, smart windows for solar control are available, which allow blocking a controllable amount of impinging solar energy. Thus, the window allows changing the solar factor and transmittance properties to adjust to outside and inside conditions, reducing the energy needed for heating and cooling. There are several working principles for this kind of adaptable windows. Firstly there are the passive ones, the photo-chromic or thermo-chromic, which change their opacity depending on the incident light or temperature conditions. As their properties change according to ambient conditions, it is not possible to implement a control system (Bertsch, Oppliger, \& Menzi, 2013). There are also active technologies, such as the gas-chromic ones, which base their opacity control on the injection of different gases in the gap between the different glass layers that compose the window (Wittwer, Graf, \& Georg, 2002). Another active technology is the electro-chromic window (ECW) (Lee et al., 2004), which reversibly changes its optical and energy transmittance properties when an external potential is applied. The ECW is the most suitable technology for architectural applications. Nevertheless, all these solutions control the day-lighting of the rooms for an improved indoor environment by rejecting most of the impinging solar energy.

As already mentioned, some facade solutions that take advantage of the incident solar energy are available on the market. Water-filled glasses are the most common solution in this group. Glass facades have very low thermal inertia, and solar radiation causes the greenhouse effect inside the buildings, causing indoor overheating and comfort decrease, forcing thus to include energy consuming air-conditioning systems. In order to limit these negative effects, some authors suggest filling the gap between the glass panes with water. This way, water blocks some solar radiation in the infrared spectrum as well as increases the thermal inertia of the building's interior, reducing the internal temperature variation and therefore, reducing the peak demand of energy. Although most of the solar energy is still entering the building, this system creates an isothermal envelope of the building to reduce the HVAC energy costs without waiving the advantages of the use of glass. Double-glazing panes with circulating hot or cold water can also be used in glass facades. Some prototypes, which can be integrated into the building's facade or roof, have been developed (www.intelliglass.es).

Another solution is the fluidised glass facade element (Gstöhl, 1998) with two fluid layers, which uses the outer liquid layer for controlling the energy transmission through the absorption of solar radiation. Despite the fact that the system unifies the functions of a shading device, solar collector and heating and cooling element, different concentrations of colorant are needed for the control of the absorptivity of the fluid. The latter requires a complex separator and a dosing feeder to be included in the system. A quite similar system was proposed later (Schwarz, 2001).

An additional related patent was previously introduced (Frantl, 1991). In this patent, a window, facade or wall construction includes at least three panes of glass, which are separated to delimit at least two intermediary spaces. A heat transfer medium, which can be coloured or pigmented, is circulated through one of the intermediary spaces. If a change in coloration or pigmentation is desired, this can be performed by means of a dosed supply, by filtering out dyes or pigments from the heat transfer medium, or by a chemical procedure that changes the $\mathrm{pH}$-value of the medium. All these solutions are difficult to be implemented and integrated in a building.

The solar active envelope module presented in this paper controls the lighting and ventilation of the room for an improved indoor environment and absorbs the part of the solar energy that is not needed inside, reducing the energy consumption of HVAC systems of the building or other applications, such as swimming pool heating. The use of solar energy is carried out through a fine and simple control of the absorptance of the whole system (Fundación Tekniker, Fundación CENER-CIEMAT, 2014). 


\section{The solar active envelope module}

Aiming to combine all the advantages of the solar active facade and solar collectors, the proposed system consists of a smart glass envelope with the ability of controlling dynamically both light and room/building temperature, taking advantage of all incident solar energy reaching the system. In the system at hand, lightness control does not imply rejecting solar energy. On the contrary, excess of solar light will be absorbed into a Heat Transfer Fluid (HTF) to improve the energy efficiency of the building.

This goal will be achieved by implementing a transparent envelope module (both roof and facade solutions are possible) that will include a HTF flowing inside. Thickness of this HTF channel will be adjusted in order to absorb the desired amount of solar light, based on the combination of the indoor user needs and the outdoor environmental conditions, and transform it into thermal energy, converting thus the building envelope into a distributed solar collector. Each facade module will be regulated independently, so it can behave as a totally transparent to totally opaque module or with any intermediate transparencies.

The proposed solar active envelope module consists of three chambers between glass panes. The fluid containing heat-absorbing nanoparticles flows inside the central chamber and is heated up due to the incident solar energy. By varying the thickness of the central chamber with a mechanical device, the absorption of the module is adjusted, as light has to cross a different fluid thickness (see Fig. 1).
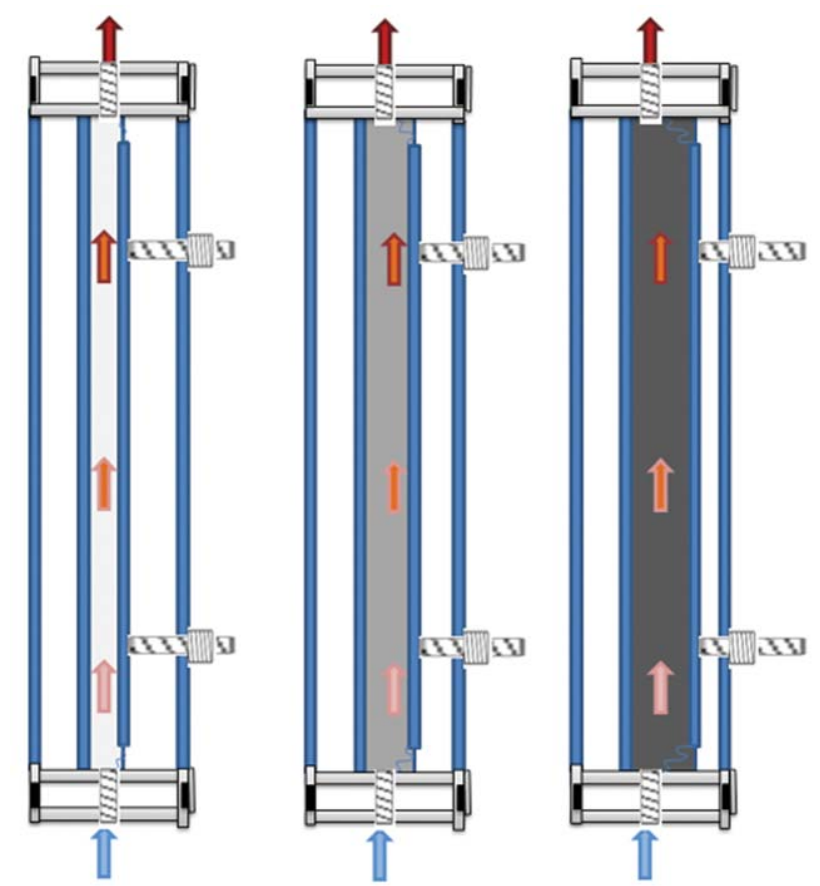

Fig. 1. Different module transparency depending on fluid thickness. NOTE: the fluid composition is always the same and so will be the transparency observed in the real cross section. The change in the color considered in this figure is just an indication of the effect of thickness adjustment in the module. 

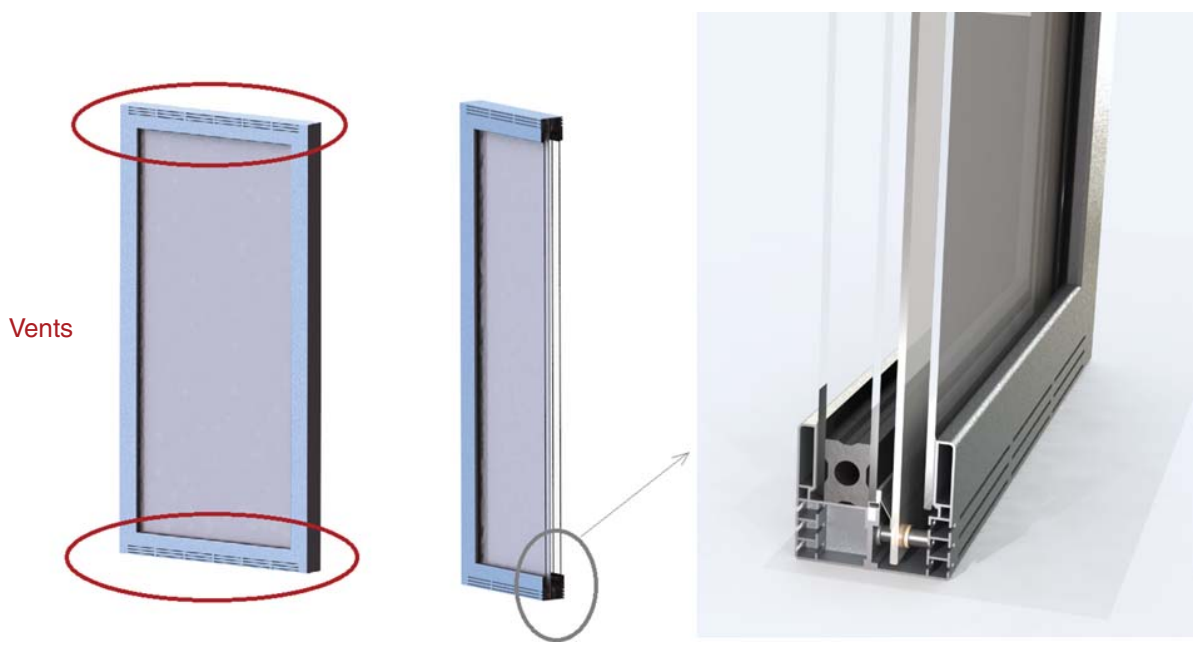

Fig. 2. Details of the concept (3D design).
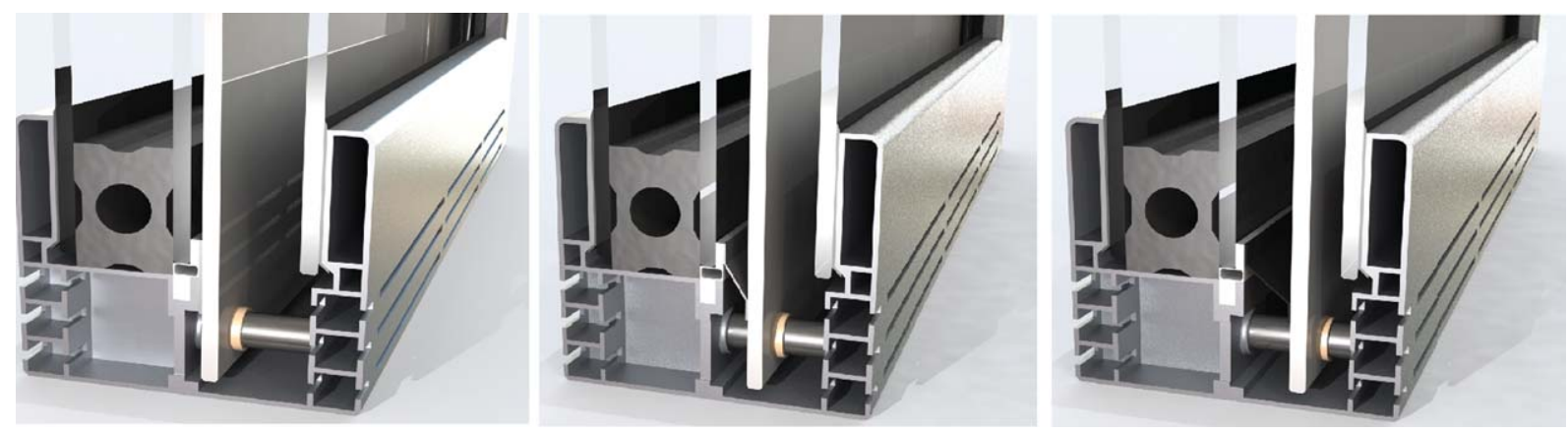

Fig. 3. The movable glass (third one from outside, left side) adjusts the thickness of the central chamber (where the fluid flows).

The thickness of the central chamber is varied with a mechanical device, which acts on one of the glasses (see Figs. 2 and 3). Depending on the position of the movable glass, the system transparency will be different, achieving as a result, a completely adjustable and automatic day lighting regulation.

Although Fig. 2 describes a system including linear actuators, a number of possible driving systems can be proposed (Fundación Tekniker, Fundación CENER-CIEMAT, 2014) including screws, pneumatic actuators, cams etc., depending on the requirements (accuracy, force, window size, space available, etc.) in each case. Water tightness is also an issue that has been taken into consideration, flexible plates or bellows must be attached to the fixed structure and to the movable glass in order to seal the whole perimeter. Moreover, glass resistance and bending due to pressure loads could be an issue that will lead to a multiple supporting points/drives design or that will limit the maximum height of a single module.

In addition, the frame of the window includes four ventilation vents, with an integrated smart filter system. Two of the ventilations vents are opened to the outside and the other two to the inside, controlling thus the indoor air-quality and comfort (see Fig. 2). 


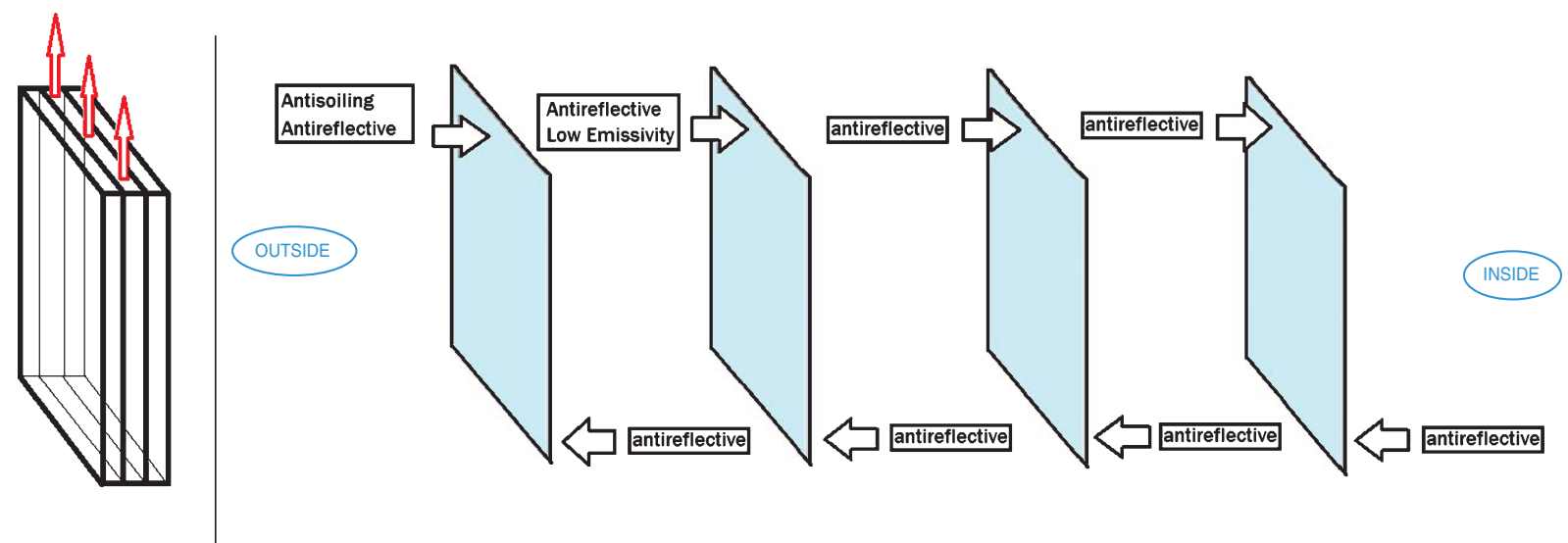

Fig. 4. Different coatings proposed for the system.

Besides, each glass layer can be covered with special coatings (see Fig. 4). Optimized optical coatings for reducing thermal losses and improving optical behaviour, and anti-soiling coatings for reducing cleaning operations, are considered in the proposed module.

\section{Operational modes of the solar active envelope module}

The proposed system is suitable to have a smart control system integrated, which will be able to dynamically adapt the system (position of the movable glass pane, flow of the fluid containing heat absorbing nanoparticles and ventilation flows) depending on the indoor needs and considering the outdoor conditions, guaranteeing thus their maximum exploitation. In this way, the system is able to:

- Act as a smart active curtain in such a way that the solar lighting is controlled by means of the thickness of the central chamber and the transparency given to the fluid. A completely adjustable and automatic daylighting regulation is achieved and indoor air-quality and comfort are guaranteed with the included ventilation vents.

- Optimize the thermal loads of the building and performance of the building envelope. As a result, the energy consumption from auxiliary heating systems and production of Domestic Hot Water (DHW) will be reduced. In addition, the thermal loads will be substantially reduced during hot days thanks to fluid blind, reducing therefore the cooling necessity and energy consumption.

- Take advantage of the energy absorbed in the fluid increasing the solar fraction of heating and cooling, reducing the thermal losses and improving the efficiency of the building. In that way, the control system could act on the system following the next foreseen procedure:

- The control system decides the lighting needs under the measured conditions and the usage pattern of the room to be controlled. Hence, it acts on the mechanical devices to adjust the thickness of the central chamber in order to obtain the required indoor illumination.

- The control system commands the mass flow inside the chamber depending on needs, being possible to absorb the excess of energy on the building envelope into the HTF as useful thermal energy. 


\section{Simulations of the proposed module}

\subsection{Methodology}

In order to characterize the performance of the active module proposed in this paper, a model of the system has been implemented and several simulations have been made. The model has been developed in a flexible, well-structured and object-oriented way, which has been accomplished applying the Modelica modeling language.

Modelica is a multi-purpose physical system modeling language and has been developed in the frame of an international initiative in order to unify already existing similar modeling approaches and to enable developed models and model libraries to be easily exchanged. The concept is based on non-causal models featuring true ordinary differential and algebraic equations, i.e. differentialalgebraic equation (DAE) systems (Elmqvist \& Mattsson, 1997). The object-oriented approach, the possibility of multiple inheritance and the re-declaration feature lead to a clear model structure, avoid multiple definitions of frequently used code and offer an incredible model flexibility. The code syntax and application guidelines are defined in the regularly updated Modelica Language Specification (https://www.modelica.org). Furthermore, the use of Modelica clearly decouples the modeler from the equation system solving. Instead of developing a specific solving algorithm for each modeling task, the Modelica tool reads the developed Modelica code, performs symbolic manipulations of equations and translates the Modelica model into a numerical simulation code, using state-of-the-art algorithms developed for general application. Thus, developed models and model libraries are exchangeable, i.e. can be read and simulated using different Modelica environments. Today, commercial, as well as open-source Modelica environments are available.

The system module model has been implemented, splitting the system in several submodels. By dividing the whole facade module into model subunits, categorized by the three basic modes of heat transfer, conduction, convection and radiation, many already existing freely available standard model structures and components could be used. Additionally, Modelica's object-oriented way of modeling led to a clear model structure and maximized the reusability of a newly developed code for future applications.

From the outside to the inside of the building, the facade module model has been divided in the following subunits (see Fig. 5 ) in order to compute temperatures (T) and mass flows (m):

- Natural convection representing the outside atmospheric conditions (Tout).

- Double glazed window (Tw) representing the first chamber and the corresponding two glass panes facing the exterior.

- An absorption fluid flow (Tff) representing the fluid with nanoparticles flowing through the middle chamber and absorbing solar energy.

- A glass pane ( $\mathrm{Tlg})$ with heat conduction.

- An air flow (Taf) representing the air flowing through the chamber facing the interior and ventilation vents.

- A glass pane (Trg) with heat conduction.

- Natural convection representing the indoor conditions (Tin).

As can be seen in Fig. 5, each module (e.g. a window) is split in $n$ nodes in order to resolve the equations. As an example, the temperature ( $T$ ) of each node and the absorption fluid flow mass flow (mff) have been obtained for two clear-sky days in a specific location assuming the indoor and outdoor conditions. 


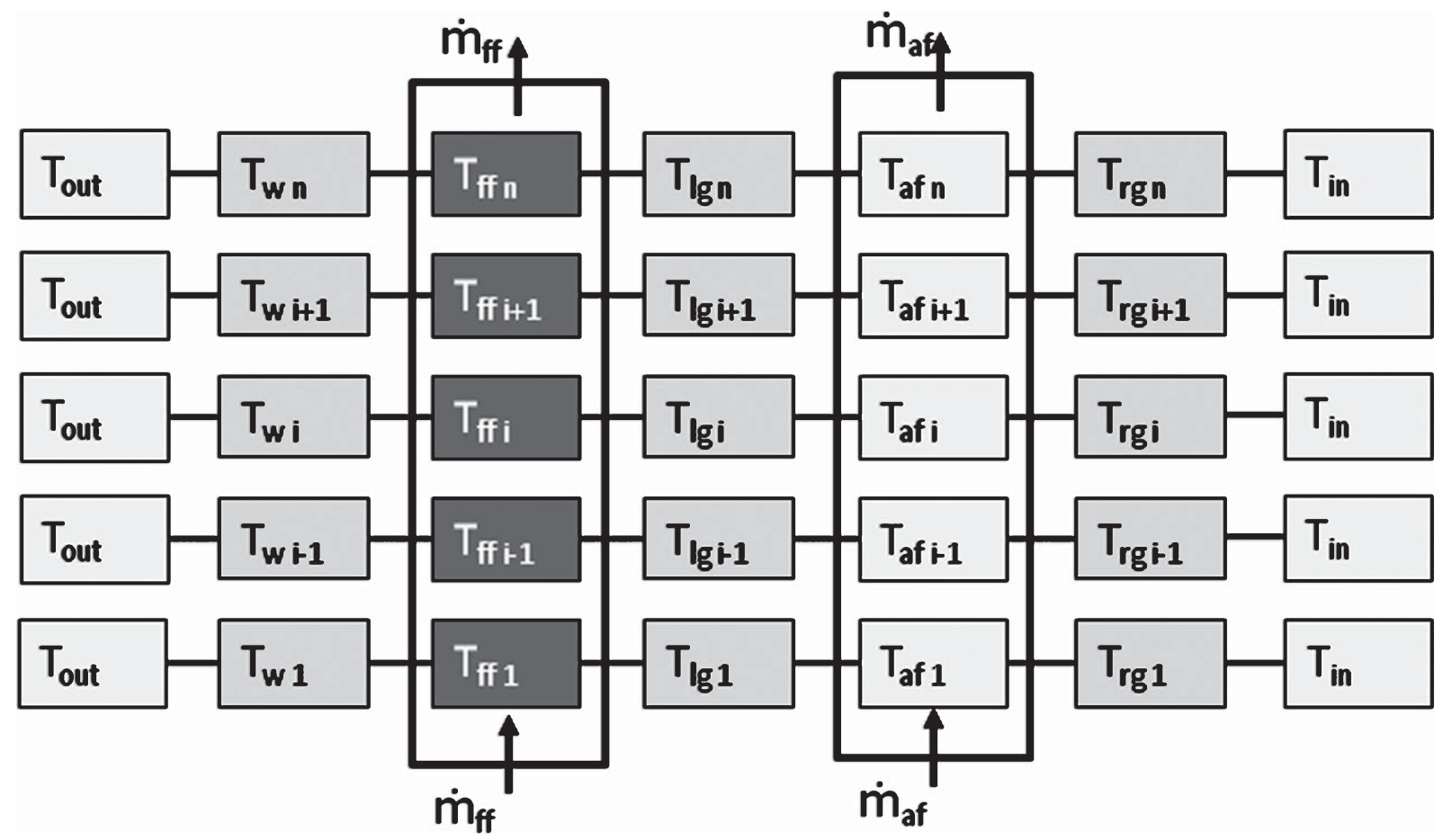

Fig. 5. System model scheme and related implemented sub-models corresponding to a single module.

The specific location which has been chosen for this analysis is San Sebastián $\left(43.32^{\circ} \mathrm{N}, 1.98^{\circ} \mathrm{W}\right)$ located in the north of Spain. An hourly clear sky year has been created and, after that, two representative days have been selected for the simulations. The selected days are the 21st of June and the 21st of December. The incident solar irradiance is needed as an input in the system model and, therefore, the impinging irradiance to a vertical facade module facing South had to be calculated from the clear-sky hourly values.

Some assumptions have to be made for the simulations. In the first place, the outdoor ambient temperature has been selected from a typical meteorological year for the location of San Sebastián. An indoor temperature of $24^{\circ} \mathrm{C}$ and an air flow of $0.1 \mathrm{~kg} / \mathrm{s}$ through ventilation vents have been assumed. For the fluid flow absorbing solar energy an inlet temperature of $35^{\circ} \mathrm{C}$, and outlet temperature of $70^{\circ} \mathrm{C}$ and an absorptance of 0.8 have been supposed.

\subsection{Results}

Using the implemented modular system model some simulations have been carried out for the specific location of San Sebastián on the 21st of June and the 21st of December. The indoor and outdoor ambient temperatures and the absorbing fluid flow inlet and outlet temperatures have been imposed, so the results of the simulation are the temperature of each node of every subsystem and the mass flow of the absorbing fluid flow. The results of the simulations corresponding to hours with impinging solar radiation are presented in the appended tables. 
Table 1

Simulation results of the proposed facade module for the 21st of June in San Sebastián

\begin{tabular}{|c|c|c|c|c|c|c|}
\hline Month & Day & Hour & $\begin{array}{l}\text { Direct Normal } \\
\text { Irradiance clear } \\
\text { sky }\left(\mathrm{W} / \mathrm{m}^{2}\right)\end{array}$ & $\begin{array}{l}\text { Irradiance impinging } \\
\text { Module }\left(\mathrm{W} / \mathrm{m}^{2}\right)\end{array}$ & $\begin{array}{l}\text { T out } \\
\left({ }^{\circ} \mathrm{C}\right)\end{array}$ & $\begin{array}{c}\dot{\mathrm{m}}_{\mathrm{ff}} \\
\left(\mathrm{kg} / \mathrm{h} \mathrm{m} \mathrm{m}^{2}\right)\end{array}$ \\
\hline 6 & 21 & 9 & 746 & 102 & 27.3 & 1.20 \\
\hline 6 & 21 & 10 & 803 & 194 & 28.6 & 2.59 \\
\hline 6 & 21 & 11 & 837 & 261 & 29.8 & 3.65 \\
\hline 6 & 21 & 12 & 854 & 290 & 30.7 & 4.08 \\
\hline 6 & 21 & 13 & 857 & 279 & 31.5 & 3.96 \\
\hline 6 & 21 & 14 & 844 & 227 & 32 & 3.12 \\
\hline 6 & 21 & 15 & 816 & 142 & 32.3 & 1.75 \\
\hline \multirow[t]{2}{*}{6} & 21 & 16 & 767 & 37 & 32.3 & 0.22 \\
\hline & & & & & Total & $20.57 \mathrm{~kg} /$ day $\mathrm{m}^{2}$ \\
\hline
\end{tabular}

Table 2

Simulation results of the proposed facade module for the 21st of December in San Sebastián

\begin{tabular}{|c|c|c|c|c|c|c|}
\hline Month & Day & Hour & $\begin{array}{l}\text { Direct Normal } \\
\text { Irradiance clear } \\
\text { sky }\left(\mathrm{W} / \mathrm{m}^{2}\right)\end{array}$ & $\begin{array}{l}\text { Irradiance impinging } \\
\text { Module }\left(\mathrm{W} / \mathrm{m}^{2}\right)\end{array}$ & $\begin{array}{l}\text { T out } \\
\left({ }^{\circ} \mathrm{C}\right)\end{array}$ & $\begin{array}{c}\dot{\mathrm{m}}_{\mathrm{ff}} \\
\left(\mathrm{kg} / \mathrm{h} \mathrm{m} \mathrm{m}^{2}\right)\end{array}$ \\
\hline 12 & 21 & 9 & 379 & 274 & 9.1 & 3.60 \\
\hline 12 & 21 & 10 & 596 & 492 & 11.1 & 7.20 \\
\hline 12 & 21 & 11 & 700 & 625 & 12.9 & 9.53 \\
\hline 12 & 21 & 12 & 745 & 684 & 14.4 & 10.56 \\
\hline 12 & 21 & 13 & 749 & 675 & 15.5 & 10.32 \\
\hline 12 & 21 & 14 & 714 & 601 & 16 & 9.12 \\
\hline 12 & 21 & 15 & 625 & 467 & 15.8 & 6.86 \\
\hline \multirow[t]{2}{*}{12} & 21 & 16 & 441 & 273 & 14.8 & 3.67 \\
\hline & & & & & Total & $65.42 \mathrm{~kg} /$ day $\mathrm{m}^{2}$ \\
\hline
\end{tabular}

As it can be seen in Table 1, a total of $20.57 \mathrm{~kg}$ per day and per $\mathrm{m}^{2}$ of facade module of fluid at $70^{\circ} \mathrm{C}$ is expected to be obtained in this location, the 21 st of June being a clear sky day. As it can be seen in Table 2, a total of $65.42 \mathrm{~kg}$ per day and per $\mathrm{m}^{2}$ of facade module of fluid at $70^{\circ} \mathrm{C}$ is expected to be obtained in this location the 21st of December being a clear sky day.

One major advantage of the proposed system in this paper is that those quantities of fluid heated at $70^{\circ} \mathrm{C}$, obtained as a result of controlling the indoor illumination, will be used for the production of DHW and for HVAC if the building is equipped with an absorption machine. In addition, the blocking part of the incoming solar radiation will contribute to reduce even more the building thermal loads decreasing the cooling needs and energy consumption.

\section{Conclusions}

This paper proposes a solar active envelope module, with a high degree of flexibility. Aiming to combine all the advantages of the solar active facade and solar collectors, the proposed system 
consists of a smart glass envelope with the ability of dynamically controlling both light and room/building temperature, taking advantage of all incident solar energy reaching the system.

The proposed solar active envelope module consists of three chambers between glass panes. The fluid containing heat absorbing nanoparticles flows inside the central chamber and is heated up due to the incident solar energy. By varying the thickness of the central chamber with a controlled mechanical device, the absorption of the module is adjusted, as light has to cross a different fluid thickness. The module transparency is based on the combination of the indoor user needs and the outdoor environmental conditions.

The proposed system is suitable to have integrated a smart control system which will be able to dynamically adapt the system. As a result, the module will:

- Act as a smart active curtain.

- Optimize the thermal loads of the building and performance of the building envelope.

- Reduce the thermal losses and improve the efficiency of the building.

In order to characterize the performance of the proposed active module a model of the system has been implemented using Modelica language. The system module model has been implemented splitting the system in several subunits.

Using the implemented module system model some simulations have been carried out for the specific location of San Sebastián the 21st of June and the 21st of December, obtaining the temperature of each node of every subsystem and the mass flow of the absorbing fluid flow.

The results of the simulations show that, in the location of San Sebastián, a total of $20.57 \mathrm{~kg}$ per day and per $\mathrm{m}^{2}$ of facade module of fluid at $70^{\circ} \mathrm{C}$ is expected to be obtained on the 21st of June being a clear sky day. In addition, a total of $65.42 \mathrm{~kg}$ per day and per $\mathrm{m}^{2}$ of facade module of fluid at $70^{\circ} \mathrm{C}$ is expected to be obtained in this location on the 21 st of December being a clear sky day. Those quantities of fluid heated at $70^{\circ} \mathrm{C}$, obtained as a result of controlling the indoor illumination, will be used for the production of DHW and for HVAC if the building is equipped with an absorption machine. In addition, the blocking part of the incoming solar radiation will contribute to a even higher reduction of the building's thermal loads decreasing the cooling needs and energy consumption.

\section{References}

Bertsch, S., Oppliger, D., \& Menzi, T. (2013). U.S. Patent No. 20130263535Al.

Elmqvist, H., \& Mattsson, S. E. (1997). Modelica - The next generation modeling language - An international design effort, Proceedings of the 1st World Congress on System Simulation, Singapore.

Frantl, E. (1991). U.S. Patent No. US4993235.

Fundación Tekniker, Fundación CENER-CIEMAT (2014). Spanish Patent No. P201431102. OEPM Madrid, Spain.

Gstöhl, D. (1998). World Organization Patent No. WO 98/51973.

Intelliglass: www.intelliglass.es/ (accessed 28.03.2015)

Lee, E.S. et al. (2004). The Energy Saving Potential of Electrochromic Windows in the US Commercial Building Modelica-Association, (2012). Modelica $^{\circledR}$ - A Unified Object-Oriented Language for Systems Modeling - Language Specification - Version 3.3, Modelica-Association, https://www.modelica.org (accessed 28.03.2015)

Schwarz, D. (2001). U.S. Patent No. US6216688.

Sector Building. Technologies Program, Environmental Energy Division, Lawrence Berkeley National Laboratory, 2004.

Wittwer, V., Graf, W., \& Georg, A. (2002). Gaschromic Glazings with a Large Dynamic Range in Total Solar Energy Transmittance. NREL 5th International Meeting on Electrochromism, 2002. 